\title{
Dor, DORT e doenças cardiovasculares em profissionais da SAMU 192 Porto Alegre/RS
}

\section{Pain, cumulative trauma disorders and cardiovascular disease in professional of SAMU 192 Porto Alegre/RS}

\author{
Fabiana de Oliveira Chaise', Tássia Silveira Furlanetto', Cláudia Tarragô \\ Candotti $^{1}$, Ana Paula Kasten ${ }^{1}$, Adriane Vieira ${ }^{1}$, Luciana Laureano Paiva ${ }^{1}$ \\ doi: http://dx.doi.org/10.11606/issn.2238-6149.v29i3p204-214
}

\begin{abstract}
Chaise FO, Furlanetto TS, Candotti CT, Kasten AP, Vieira A, Paiva LL. Dor, DORT e doenças cardiovasculares em profissionais da SAMU 192 Porto Alegre/RS. Rev Ter Ocup Univ São Paulo. 2018 set.-dez.;29(3):204-14.
\end{abstract}

RESUMO: Objetivo: identificar a prevalência de dor musculoesquelética, distúrbios osteomusculares relacionadas ao trabalho (DORT), doenças cardiovasculares (DCV) e os fatores associados em profissionais do SAMU 192 Porto Alegre/RS. Métodos: 113 trabalhadores (37\% da equipe do SAMU 192 Porto Alegre/ $\mathrm{RS}$ ) responderam a um questionário de hábitos relacionados ao trabalho, riscos ocupacionais e o aparecimento de DCV e DORT. A análise estatística foi realizada com tabelas de frequência, Teste Qui-quadrado e Razões de Prevalência (IC 95\%) por meio de análise multivariável (modelo de Regressão de Poisson). $\alpha=0,05$. Resultados: Todos participantes estavam na condição de sobrepeso e apresentaram alta prevalência de dor $(92,9 \%)$ e DORT $(50,4 \%)$ e baixa prevalência de DCV $(8,8 \%)$. A idade atuou como um aumento no risco para o aparecimento da dor. Os fatores associados para o aparecimento da DORT foram a exposição ao frio, ao calor e às vibrações, esforço físico, ausência de pausa nas atividades, profissão, regime de trabalho e estresse. A associação entre os fatores associados e DCV não foi possível devido à baixa prevalência de DCV entre os trabalhadores. Conclusão: os profissionais do SAMU 192 Porto Alegre/RS apresentaram alta prevalência de dor e DORT e baixa prevalência de DCV.

Descritores: Serviços médicos de emergência; Dor; Transtornos traumáticos cumulativos; Doenças cardiovasculares.

\begin{abstract}
Chaise FO, Furlanetto TS, Candotti CT, Kasten AP, Vieira A, Paiva LL. Pain, cumulative trauma disorders and cardiovascular disease in professional of SAMU 192 Porto Alegre/RS. Rev Ter Ocup Univ São Paulo. 2018 Sept.-Dec.;29(3):204-14.

ABSTRACT: Objective: To determine the prevalence of musculoskeletal pain, Cumulative Trauma Disorders (CTD) and cardiovascular disease (CVD) and identify the associated factors in the emergency professionals of Porto Alegre/RS. Methods: 113 workers (37\% of SAMU 192 Porto Alegre/RS) answered a questionnaire about work-related habits, occupational risks and the onset of CTD and CVD. Statistical analysis was performed using frequency tables, chi-square test and prevalence ratios (95\%) through multivariate analysis (Poisson's regression model). Considering $\alpha=0.05$. Results: All participants were in the overweight condition and showed high prevalence of pain $(92.9 \%)$ and CTD (50.4\%), and low CVD prevalence (8.8\%). Age acted as a risk for pain. Factors associated with CTD were exposure to cold and heat, exposure to vibrations, physical exertion, no breaks in between activities, occupation, working hours and stress. The association between risk and CVD was not possible due to the low prevalence of CVD among workers. Conclusion: Samu 192 professionals of Porto Alegre/RS showed high prevalence of pain and CTD, and low prevalence of CVD.
\end{abstract}

Keywords: Emergency medical services; Pain; Cumulative trauma disorders; Cardiovascular diseases.

O presente estudo foi parte integrante da dissertação de mestrado "Fatores de riscos à saúde cardiovascular e osteomuscular relacionados aos hábitos, estilo de vida e ao trabalho em profissionais do SAMU 192 Porto Alegre/RS", de Fabiana de Oliveira Chaise, Programa de Pós Graduação em Ciências do Movimento Humano, UFRS, em 2015.

1. Universidade Federal do Rio Grande do Sul. E-mail: fabianachaise@gmail.com, tassiasf@gmail.com, claudia.candotti@ufrgs.br, anapaulakasten@hotmail.com, adriane.vieira@gmail.com, lucianalaureanopaiva@gmail.com.

Endereço para correspondência: Tássia Silveira Furlanetto. Rua Albino Paul, 45. Bairro Jardim América, São Leopoldo, RS. CEP: 93032-280. E-mail: tassiasf@gmail.com. 


\section{INTRODUÇÃO}

$\mathrm{O}$ atendimento pré-hospitalar às urgências consiste em toda e qualquer assistência realizada, direta ou indiretamente, fora do ambiente hospitalar, visando à manutenção da vida e/ou a minimização das sequelas ${ }^{1}$. No Brasil, este tipo de serviço é denominado de Serviço de Atendimento Móvel de Urgência (SAMU 192), sendo normatizado em 2003 para todo o território brasileiro ${ }^{2}$. Atualmente 123.429.882 milhões de habitantes têm acesso ao SAMU 192 no país, o que corresponde a uma cobertura de $65 \%$ da população brasileira ${ }^{3}$.

Risco é considerado qualquer perigo, inconveniência, dano ou fatalidade eventual, provável ou previsível. O conceito de risco à saúde do trabalhador representa a possibilidade de ocorrer um efeito adverso ou dano ao seu bem-estar ou ao da comunidade ${ }^{4}$. Os trabalhadores do serviço de urgência estão expostos a diversos riscos ocupacionais, podendo ser oriundos de agentes físicos (como ruídos e vibrações), químicos (como poeira e gases), biológicos (como vírus e bactérias), acidentais (como equipamentos sem proteção e probabilidade de acidente automobilístico) e ergonômicos (condições ambientais de trabalho, como esforço físico intenso e levantamento e transporte de peso; e a organização do trabalho, como o ritmo de trabalho e inexistência de pausas) ${ }^{5}$.

Além desses, existem os riscos psicossociais, os quais englobam as relações sociais entre e no ambiente de trabalho, bem como as percepções e experiências subjetivas dos trabalhadores em relação ao ambiente de trabalho e ao trabalho realizado ${ }^{6,7}$. Os riscos psicossociais produzem sobrecarga psíquica ${ }^{4}$, portanto sua avaliação leva em conta o nível de estresse e seus desencadeantes, além da vivência do trabalhador em relação à sua insatisfação no trabalho ${ }^{7}$. Comumente, esses riscos estão contemplados dentro do grupo de riscos ergonômicos ${ }^{4}$.

O estresse, por sua vez, é um quadro onde existe uma desproporção prolongada entre o grau de tensão do indivíduo e a sua capacidade de suportá-lo, podendo desencadear distúrbios emocionais ${ }^{8}$. Em outras palavras, o estresse é o que acontece quando as pessoas enfrentam situações onde seu conjunto de comportamentos é insuficiente para sua adaptação, geralmente desencadeado em situações onde as demandas excedem as habilidades ${ }^{9}$. Como exemplo de fatores desencadeantes do estresse ocupacional, estão: insatisfação no trabalho, sobrecarga de trabalho, processo de tomada de decisão e pressão excessiva ${ }^{8,9}$.

$\mathrm{O}$ aparecimento dos distúrbios osteomusculares relacionadas ao trabalho (DORT) é muito comum em trabalhadores do serviço de emergência. DORT pode ser definida como:

\begin{abstract}
"Considerados transtornos que apresentam como características comuns o aparecimento e a evolução de caráter insidioso, origem multifatorial complexa, na qual se entrelaçam inúmeros fatores causais, entre eles exigências mecânicas repetidas por períodos de tempo prolongados, utilização de ferramentas vibratórias, posições forçadas, fatores da organização do trabalho, como, por exemplo, exigências de produtividade, competitividade, programas de incentivo à produção e de qualidade. Essas utilizam estratégias de intensificação do trabalho e de controle excessivo dos trabalhadores, sem levar em conta as características individuais do trabalhador, os traços de personalidade e sua história de vida" (p. 425) ${ }^{10}$.
\end{abstract}

Os sinais e sintomas dos DORT são diversos, tais como: dor espontânea ou ao movimento; sensações de fraqueza, cansaço, dormência e/ou agulhadas; e dificuldade para utilização dos membros em atividades cotidianas. Além disso, é preciso investigar cuidadosamente a localização, intensidade, maneira e momento de início das queixas, duração e caracterização do tempo de evolução e identificação dos fatores que contribuem para a melhora ou agravamento do quadro ${ }^{10}$.

Uma das principais causas para o aparecimento dos DORT é a exposição excessiva aos riscos ergonômicos ${ }^{11,12}$. Mesmo assim, outros fatores podem estar relacionados também ao aparecimento de DORT. Já as doenças cardiovasculares (DCV) estão relacionadas, sobretudo, à história familiar precoce de DCV, obesidade, sedentarismo, etnia e aos riscos psicossociais ${ }^{13}$.

Assim, para dar conta da demanda de trabalho e do fluxo de atendimento, o trabalhador da SAMU 192 necessita alguns pré-requisitos, como equilíbrio emocional e autocontrole, condicionamento físico para a função, capacidade de trabalhar em equipe e disposição para cumprir ações orientadas ${ }^{2}$. Além disso, o contato com o sofrimento e a morte de pacientes e os riscos a que são expostos durante o serviço de atendimento podem influenciar de forma negativa a saúde mental desses trabalhadores, podendo desencadear o estresse ${ }^{14}$.

O contexto dos trabalhadores do SAMU 192 Porto Alegre/RS enquadra-se no contexto internacional dos trabalhadores do serviço de emergência, e, até onde se tem conhecimento, não há estudos que identifiquem pontualmente a realidade de saúde desses trabalhadores. Assim, o objetivo do presente estudo foi identificar a prevalência de dor musculoesquelética, DORT e DCV e identificar os fatores associados em profissionais do SAMU 192 Porto Alegre/RS.

\section{METODOLOGIA}

Este é um estudo descritivo, transversal e de base 
populacional, em que participaram trabalhadores de ambos os sexos do SAMU 192 Porto Alegre/RS. Inicialmente, foi apresentado ao coordenador geral do serviço préhospitalar do SAMU 192 o projeto desse estudo buscando a autorização e colaboração. Em um segundo momento, após o interesse demonstrado pelo coordenador, buscou-se a autorização Secretaria Municipal de Saúde para realização do estudo. Portanto, esse estudo contou com a autorização e cooperação da Secretaria Municipal de Saúde do município de Porto Alegre, RS.

O contato inicial com a realidade do trabalhador do SAMU 192 foi durante o período da residência multiprofissional em saúde de uma das pesquisadoras. Esse estudo é proveniente do projeto de mestrado dessa pesquisadora, posterior ao período da residência. Esse fator foi um elo desencadeador da motivação desse estudo, aliado ao fato do estudo ter sido conduzido em um grupo de pesquisa que investiga a dor musculoesquelética e a postura corporal.

O período de realização do procedimento de coleta dos dados foi entre março e dezembro de 2013. O convite para os trabalhadores foi realizado pessoalmente pelos pesquisadores em cada uma das bases descentralizadas do SAMU 192. Esse estudo foi conduzido dentro dos padrões éticos exigidos pela Comissão Nacional de Ética em Pesquisa/Conselho Nacional de Saúde/Ministério da Saúde (CONEP/CNS/MS), aprovado pelo Comitê de Ética em Pesquisa da Universidade onde foi realizado sob o $\mathrm{n}^{\mathrm{o}}$ 13186413.4.3001.5338 e todos os trabalhadores consentiram em participar do estudo voluntariamente.

O quadro funcional do SAMU 192 é composto por condutores de ambulância, técnicos de enfermagem, enfermeiros, médicos reguladores e telefonistas auxiliares de regulação médica (TARM). No presente estudo, todos os profissionais foram convidados a participar, sendo que aqueles que aceitaram o convite foram agrupados, para fins de análise, em: Equipe Móvel (condutor de ambulância e técnico de enfermagem) e Equipe Fixa (enfermeiro, médico regulador e TARM). Foram excluídos do estudo funcionários que estavam afastados por motivo de doença ou licença maternidade durante o período de coleta de dados e os que não consentiram em participar da pesquisa.

Para a coleta de dados foi utilizado um questionário de hábitos relacionados ao Trabalho (QHT), o qual teve confirmada sua validade de conteúdo e reprodutibilidade em estudo prévio ${ }^{15}$. O QHT é um instrumento composto por questões abertas e fechadas que tem por objetivo investigar o universo do trabalho nos aspectos jornada de trabalho, riscos ocupacionais, estresse no ambiente de trabalho e o aparecimento de DCV e DORT.
Do QHT foram extraídos dados relacionados a três núcleos: DCV, dor musculoesquelética e DORT. Em relação à presença ou não de DCV e de DORT, o estudo baseou-se exclusivamente na resposta dos participantes da pesquisa e não em dados clínicos ou em exames. Do núcleo das DCV foi investigado o auto relato dessas doenças e os fatores associados, como o estresse, oriundo da organização do trabalho, o tabagismo, o etilismo, o sedentarismo, a obesidade, a hipertensão arterial sistêmica (HAS) e o Diabetes Melito (DM). Do núcleo dor musculoesquelética e DORT foram investigadas as regiões de dor, frequência e intensidade nos últimos três meses, as DORT apresentadas e os fatores associados, tais como: a organização do trabalho, os fatores ambientais e as possíveis sobrecargas de segmentos corporais.

Cada trabalhador do SAMU 192 Porto Alegre/ RS recebeu o QHT impresso juntamente com o Termo de Consentimento Livre e Esclarecido (TCLE) em mãos dos próprios pesquisadores. Em um período máximo de sete dias os questionários preenchidos deveriam ser entregues pelos funcionários aos pesquisadores. Foi orientado aos trabalhadores que o preenchimento do questionário deveria ser realizado individualmente e com respostas fiéis aos questionamentos, sem comunicação entre os participantes da pesquisa.

As respostas dos questionários foram codificadas e tabuladas, sendo submetidas às análises estatísticas no software SPSS versão 18. Foi utilizada estatística descritiva, por meio de tabelas de frequência para a descrição do perfil dos trabalhadores. O Teste do Qui-quadrado foi utilizado para verificar as associações entre os fatores associados para as DCV. Foram também calculadas as Razões de Prevalência (RP) e seus respectivos Intervalos de Confiança de 95\% (IC 95\%) por meio de análise multivariável realizada a partir do modelo de Regressão de Poisson. O nível de significância adotado foi $\alpha=0,05$.

\section{RESULTADOS}

De um total de 300 questionários entregues, 113 retornaram aos pesquisadores. Portanto, para fins de análise, a população estudada correspondeu a 113 trabalhadores, que representa $37 \%$ do total de trabalhadores do SAMU 192 Porto Alegre/RS.

\section{Doenças Cardiovasculares (DCV)}

O perfil sociodemográfico dos trabalhadores do SAMU 192 indicou um predomínio de trabalhadores do sexo masculino $(63,6 \%)$ na equipe móvel e do sexo feminino $(72,2 \%)$ na equipe fixa. Ambas as equipes apresentaram um 
IMC $>25 \mathrm{~kg} / \mathrm{m} 2$ considerado pela Organização Mundial da Saúde (OMS) como sendo sobrepeso (Tabela 1). A maioria dos trabalhadores $91,2 \%(\mathrm{n}=103)$ não apresentou $\mathrm{DCV}$, sendo citadas por $8,8 \%(\mathrm{n}=10)$ as doenças: insuficiência cardíaca, arritmia cardíaca, infarto e acidente vascular cerebral (AVC).

Tabela 1 - Perfil sociodemográfico dos trabalhadores do SAMU 192 Porto Alegre/RS, divididos por equipes móvel e fixa

\begin{tabular}{lcc}
\hline & $\begin{array}{c}\text { Equipe móvel } \\
(\mathrm{n}=77)\end{array}$ & $\begin{array}{c}\text { Equipe fixa } \\
(\mathrm{n}=36)\end{array}$ \\
\hline Sexo \% (n) & & $27,8 \%(10)$ \\
Masculino & $63,6 \%(49)$ & $72,2 \%(26)$ \\
Feminino & $36,4 \%(28)$ & $39,4( \pm 9,3)$ \\
Idade (anos) (dp) & $42,5( \pm 9,2)$ & $1,63( \pm 8,3)$ \\
Estatura (m) (dp) & $1,7( \pm 9,2)$ & $77,6( \pm 19,7)$ \\
Massa corporal (kg) (dp) & $79,4( \pm 17,4)$ & $27,8( \pm 7,5)$ \\
IMC (kg/m²) & $26,9( \pm 5,2)$ & \\
Escolaridade \% (n) & & \\
Ensino Fundamental Completo & $10,4 \%(8)$ & $25 \%(9)$ \\
Ensino Fundametal Incompleto & $5,2 \%(4)$ & $5,6 \%(2)$ \\
Ensino Médio Completo & $42,9 \%(33)$ & $13,9 \%(5)$ \\
Ensino Médio Incompleto & $5,2 \%(2)$ & $5,6 \%(2)$ \\
Ensino Superior Completo & $10,4 \%(8)$ & $50 \%(18)$ \\
Ensino Superior Incompleto & $22,1 \%(17)$ & \\
Pós-Graduação & $3,9 \%(3)$ & \\
Pós-Graduação em andamento & & \\
\hline
\end{tabular}

Entre os trabalhadores, foram identificados fatores associados modificáveis para as DCV (tabagismo, sedentarismo, IMC, HAS, DM e estresse), não modificáveis (hereditariedade para doença coronariana e idade), ergonômicos (regime de trabalho, horas extras), além de considerar o histórico ocupacional do tempo de serviço como um fator associado. A associação entre os fatores associados e DCV não foi possível devido à baixa prevalência de DCV entre os trabalhadores. Assim foram realizadas associações entre os próprios fatores associados, mas somente o fator HAS apresentou algumas associações significativas com outros fatores (Tabela 2).

Foi encontrada associação significativa entre HAS e IMC, idade e tempo de serviço, onde as razões de prevalência mostram que quem estava na condição de sobrepeso tinha $13 \%$ a mais de probabilidade de apresentar HAS do que quem estava na condição normal. Quanto à idade, quem estava acima dos 40 anos tinha $22 \%$ a mais de probabilidade de apresentar HAS do que quem estava abaixo desta faixa etária. E, quanto ao histórico ocupacional do tempo de serviço, os trabalhadores com 11 anos de serviço ou mais, tinham $15 \%$ a mais de probabilidade de apresentar HAS do que os demais trabalhadores (Tabela 2).

\section{Dor e DORT}

Os resultados do estudo indicaram que o regime de trabalho dos integrantes da equipe móvel (100\%) era de plantão de $12 \mathrm{hs}$, e da equipe fixa, em sua maioria (72,2\%), era de menos de 12 horas diária. Para ambas as equipes a maioria dos trabalhadores estava na faixa de até 10 anos de serviço, realizava horas extras mensais e não possuía outro vínculo empregatício. Quanto aos acidentes de trabalho, a equipe móvel apresentava maior frequência para mais de um tipo de acidente, enquanto que a equipe fixa apresentava maior frequência para um único tipo de acidente (Tabela 3), que é a agressão física e moral. 
Chaise FO, et al. Dor, DORT e doenças cardiovasculares em profissionais. Rev Ter Ocup Univ São Paulo. 2018 set.-dez.;29(3):204-14.

Tabela 2 - Fatores associados à Hipertensão Arterial Sistêmica (HAS), teste do Qui-quadrado $\left(\mathrm{x}^{2}\right)$ e as razões de prevalência dos trabalhadores do SAMU 192 Porto Alegre/RS

\begin{tabular}{|c|c|c|c|c|}
\hline \multirow[b]{2}{*}{ Fatores associados } & \multirow[b]{2}{*}{ N (\%) } & \multicolumn{3}{|c|}{ HAS } \\
\hline & & N (\%) & $\mathbf{x}^{2}$ & RP (IC 95\%) \\
\hline \multicolumn{5}{|l|}{ IMC } \\
\hline Normal & $41(36,3)$ & $5(4,9)$ & \multirow{2}{*}{$0,010^{*}$} & 1 \\
\hline Sobrepeso & $72(63,7)$ & $17(23,6)$ & & $1,13(0,77-1,65)$ \\
\hline \multicolumn{5}{|l|}{ Colesterol alto } \\
\hline Não & $97(85,8)$ & $18(18,6)$ & \multirow{2}{*}{0,223} & 1 \\
\hline Sim & $16(14,2)$ & $3(6,2)$ & & $1,10(0,80-1,55)$ \\
\hline \multicolumn{5}{|l|}{ Idade } \\
\hline Até 39 anos & $51(45,1)$ & $1(2,0)$ & \multirow{2}{*}{$<0,001^{*}$} & 1 \\
\hline 40 anos ou mais & $62(54,9)$ & $18(29)$ & & $1,22(0,85-1,75)$ \\
\hline \multicolumn{5}{|l|}{ Tabagismo } \\
\hline Não & $83(73,5)$ & $17(20,5)$ & \multirow{2}{*}{0,080} & 1 \\
\hline Sim & $30(26,5)$ & $5(6,7)$ & & $0,90(0,59-1,36)$ \\
\hline \multicolumn{5}{|l|}{ Sedentarismo } \\
\hline Não & $50(44,2)$ & $12(24)$ & \multirow{2}{*}{0,063} & 1 \\
\hline Sim & $63(55,8)$ & $7(11,1)$ & & $0,90(0,64-1,27)$ \\
\hline \multicolumn{5}{|l|}{ Diabetes } \\
\hline Não & $104(92)$ & $16(15,4)$ & \multirow{2}{*}{0,167} & 1 \\
\hline Sim & $9(8)$ & $5(33,3)$ & & $1,05(0,53-1,93)$ \\
\hline \multicolumn{5}{|l|}{ Hereditariedade } \\
\hline Não & $60(54,5)$ & $10(16,7)$ & \multirow{2}{*}{0,925} & 1 \\
\hline $\operatorname{Sim}$ & $50(45,5)$ & $8(16)$ & & $0,99(0,70-1,40)$ \\
\hline \multicolumn{5}{|l|}{ Tempo de Serviço } \\
\hline Até 10 anos & $82(72,6)$ & $10(12,2)$ & \multirow{2}{*}{$0,033^{*}$} & 1 \\
\hline 11 anos ou mais & $31(27,4)$ & $9(29)$ & & $1,15(0,65-1,65)$ \\
\hline Regime de Trabalh & & & & \\
\hline Menos de 12 horas & $26(23)$ & $5(15,4)$ & & 1 \\
\hline Plantão de 12 horas & $87(77)$ & $15(17,2)$ & 0,824 & $1,01(0,67-1,52)$ \\
\hline Horas extras & & & & \\
\hline Não & $19(16,8)$ & $3(10,5)$ & & 1 \\
\hline Sim & $94(83,2)$ & $17(18,1)$ & 0,422 & $1,06(0,67-1,70)$ \\
\hline Estresse na tomada & & & & \\
\hline Não exposto & $14(12,4)$ & $4(28,6)$ & مح00 & 1 \\
\hline Exposto & $99(87,6)$ & $15(15,2)$ & 0,209 & $0,91(0,47-1,76)$ \\
\hline Tempo e velocidade & & & & \\
\hline Não exposto & $9(8)$ & $2(22,2)$ & 0651 & 1 \\
\hline Exposto & $104(92)$ & $17(16,3)$ & 0,651 & $0,94(0,45-1,94)$ \\
\hline Trabalho em equip & & & & \\
\hline Não exposto & $19(16,8)$ & $5(26,3)$ & & 1 \\
\hline Exposto & $94(83,2)$ & $14(14,9)$ & 0,225 & $0,92(0,50-1,66)$ \\
\hline Relação com a chef & & & & \\
\hline Não exposto & $36(31,9)$ & $7(19,4)$ & 0600 & 1 \\
\hline Exposto & $77(68,1)$ & $12(15,6)$ & 0,609 & $1,00(0,63-1,58)$ \\
\hline Remuneração & & & & \\
\hline Não exposto & $23(20,4)$ & $4(13)$ & 0588 & 1 \\
\hline Exposto & $90(79,6)$ & $16(17,8)$ & 0,588 & $1,06(0,65-1,71)$ \\
\hline Jornada de Trabalh & & & & \\
\hline Não exposto & $28(24,8)$ & $5(17,9)$ & 0865 & 1 \\
\hline Exposto & $85(75,2)$ & $14(16,5)$ & 0,865 & $1,03(0,61-1,76)$ \\
\hline Pressão para produ & & & & \\
\hline Não exposto & $33(29,2)$ & $6(18,2)$ & 0803 & 1 \\
\hline Exposto & $80(70,8)$ & $13(16,2)$ & 0,803 & $1,03(0,64-1,66)$ \\
\hline Restrição do sono & & & & \\
\hline Não exposto & $26(23)$ & $5(19,2)$ & 0707 & 1 \\
\hline Exposto & $87(77)$ & $14(16,1)$ & 0,707 & $0,94(0,57-1,52)$ \\
\hline
\end{tabular}


Tabela 3 - Perfil ocupacional dos trabalhadores do SAMU 192 Porto Alegre/RS, divididos por equipes móvel e fixa

\begin{tabular}{|c|c|c|}
\hline & $\begin{array}{c}\text { Equipe Móvel } \\
\qquad(\mathrm{n}=77)\end{array}$ & $\begin{array}{c}\text { Equipe Fixa } \\
(n=36)\end{array}$ \\
\hline \multicolumn{3}{|l|}{ Regime de Trabalho } \\
\hline Menos de $12 \mathrm{hs}$ & & $72,2 \%(26)$ \\
\hline Plantão de 12 hs & $100 \%(77)$ & $27,8 \%(10)$ \\
\hline \multicolumn{3}{|l|}{ Turno de Trabalho } \\
\hline Manhã & & $13,9 \%(5)$ \\
\hline Tarde & & $19,4 \%(7)$ \\
\hline Noite & $33,8 \%(26)$ & $5,6 \%(2)$ \\
\hline Manhã e Tarde & $45,5 \%(35)$ & $22,2 \%(8)$ \\
\hline Alternado & $20,8 \%(16)$ & $38,9 \%(14)$ \\
\hline \multicolumn{3}{|l|}{ Tempo de Serviço } \\
\hline Até 10 anos & $67,5 \%(52)$ & $83,3 \%(30)$ \\
\hline 11 anos ou mais & $32,5 \%(25)$ & $16,7 \%(6)$ \\
\hline \multicolumn{3}{|l|}{ Horas Extras } \\
\hline Não & $6,5 \%(5)$ & $38,9 \%(14)$ \\
\hline Sim & $93,5 \%(72)$ & $61,1 \%(22)$ \\
\hline 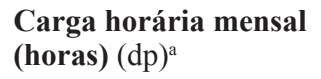 & $173,8( \pm 37,6)$ & $200,6( \pm 66,2)$ \\
\hline \multicolumn{3}{|l|}{$\begin{array}{l}\text { Outro vìnculo de } \\
\text { Trabalho }\end{array}$} \\
\hline Não & $84,4 \%(65)$ & $58,3 \%(21)$ \\
\hline Sim & $15,6(12)$ & $41,7 \%(15)$ \\
\hline \multicolumn{3}{|l|}{ Acidente de Trabalho } \\
\hline Um tipo $^{\mathbf{b}}$ & $45,5 \%(35)$ & $83,3 \%(30)$ \\
\hline Mais de um tipo ${ }^{c}$ & $54,5 \%(42)$ & $16,7 \%(6)$ \\
\hline
\end{tabular}

a Carga horária mensal incluindo as horas extras

${ }^{b}$ Tipo de acidente de trabalho: contaminações por substâncias químicas e biológicas, material perfuro-cortante, agressões físicas e morais e acidente automobilístico.

${ }^{c}$ Combinação de mais de um tipo de acidente de trabalho descritos em ${ }^{\mathrm{b}}$
A prevalência de dores musculoesqueléticas entre os trabalhadores do SAMU 192 Porto Alegre/RS foi de $92,9 \%(n=105)$. As regiões do corpo mais acometidas foram: "costas e membros inferiores (MMII)" (35,2\%), costas (33,3\%) e "costas e membros superiores (MMSS)" $(17,1 \%)$. Quanto a frequência e intensidade da dor musculoesquelética, as prevalências mais elevadas foram na região das costas $(33,3 \%, \mathrm{n}=35)$ e "costas e MMII" (35,2\%, $\mathrm{n}=37)$. Já em relação à intensidade da dor, a região das costas $(29,5 \%)$ e "costas e MMII" (31,4\%) apresentou alta prevalência de dor moderada e forte. Não houve associações significativas entre a dor e as variáveis ocupacionais, entretanto a variável sociodemográfica idade apresentou associação significativa $(\mathrm{p}=0,008)$ com a dor, de forma que dos 62 trabalhadores com idade de 39 anos ou mais, $87,1 \%$ $(n=54)$ relataram a presença de dor. Os resultados indicam que quem tinha 39 anos ou mais tinha $6 \%$ a mais de chance de ter dor musculoesquelética que os trabalhadores das demais faixas etárias.

A prevalência de DORT foi de 50,4\% ( $\mathrm{n}=57)$ nos trabalhadores da SAMU 192 Porto Alegre/RS. As DORT referidas foram: tendinite no punho (39\%), hérnia de disco $(11 \%)$, bursite $(7 \%)$ e tenossinovite $(3 \%)$.

$\mathrm{Na}$ equipe móvel, foram encontradas associações significativas $(\mathrm{p}<0,05)$ entre todos os fatores associados descritos e as DORT (Tabela 4). As razões de prevalência confirmam que tais fatores se comportam como risco aos trabalhadores. Merece destaque os riscos físicos (exposição a vibrações, como por exemplo, o ruído acentuado e constante de sirene das ambulâncias e a vibração excessiva do motor desses veículos pesados durante o transporte) e riscos ergonômicos (como por exemplo, a ausência de pausa nas atividades), pois o trabalhador que está exposto a eles apresenta $39 \%$ e $36 \%$, respectivamente, a mais de chance de desenvolver DORT do que os trabalhadores não expostos. Devido ao fato de que a equipe fixa estava menos exposta às DORT do que a equipe móvel foi considerada a ausência de risco para as DORT $(\mathrm{RP}=1)$.

As DORT também obtiveram associação significativa com a dor musculoesquelética $(\mathrm{p}=0,02)$. Entre os 105 trabalhadores que apresentavam dor, 53,3\% tinham alguma DORT. Os resultados da razão de prevalência indicam que quem tinha dor musculoesquelética, tinha $36 \%$ de chance a mais de ter DORT que os demais trabalhadores. 
Tabela 4 - Fatores associados às DORT, teste do Qui-quadrado $\left(\mathrm{X}^{2}\right)$ e as razões de prevalência da equipe móvel dos trabalhadores da SAMU 192 Porto Alegre/RS

\begin{tabular}{|c|c|c|c|c|}
\hline \multirow{2}{*}{ Fatores associados } & \multicolumn{4}{|c|}{ DORT } \\
\hline & $\mathbf{N}(\%)$ & $\mathbf{N}(\%)$ & $\mathbf{X}^{2}$ & RP (IC 95\%) \\
\hline \multicolumn{5}{|c|}{ Exposição ao calor e ao frio } \\
\hline Não expostos & $12(9,8)$ & $5(36,4)$ & \multirow{2}{*}{$0,048^{*}$} & 1 \\
\hline Expostos & $101(90,2)$ & $53(52,5)$ & & $1,036(0,59-1,81)$ \\
\hline \multicolumn{5}{|l|}{ Exposição a vibrações } \\
\hline Não expostos & $22(18,8)$ & $4(19)$ & \multirow{2}{*}{$0,010^{*}$} & 1 \\
\hline Expostos & $91(81,2)$ & $53(58,2)$ & & $1,39(0,95-1,11)$ \\
\hline \multicolumn{5}{|l|}{ Esforço físico } \\
\hline Não expostos & $15(12,5)$ & $5(35,7)$ & \multirow{2}{*}{$0,018^{*}$} & 1 \\
\hline Expostos & $98(87,5)$ & $52(53,1)$ & & $1,19(0,73-1,95)$ \\
\hline \multicolumn{5}{|c|}{ Pausa nas tarefas/atividades } \\
\hline Nunca & $99(87,5)$ & $55(56,1)$ & \multirow{2}{*}{$0,003 *$} & $1,36(0,81-2,28)$ \\
\hline Sempre & $14(12,5)$ & $5(14,3)$ & & 1 \\
\hline \multicolumn{5}{|l|}{ Profissão } \\
\hline Condutores e Técnicos & $76(67,9)$ & $44(57,9)$ & \multirow{2}{*}{$0,030^{*}$} & $1,16(0,83-1,61)$ \\
\hline Enf., Médicos e TARM & $36(32,1)$ & $13(36,1)$ & & 1 \\
\hline \multicolumn{5}{|l|}{ Regime de Trabalho } \\
\hline Menos de 12 hs & $26(23,2)$ & $9(34,6)$ & \multirow{2}{*}{$0,048^{*}$} & 1 \\
\hline Plantão de 12 hs & $87(76,8)$ & $48(55,8)$ & & $1,15(0,79-1,67)$ \\
\hline \multicolumn{5}{|c|}{ Estresse tempo e velocidade da realização da tarefa } \\
\hline Não expostos & $12(8)$ & $7(22,2)$ & \multirow{2}{*}{$0,070^{*}$} & 1 \\
\hline Expostos & $101(92)$ & $56(53,4)$ & & $1,25(0,68-2,30)$ \\
\hline
\end{tabular}

*associação significativa

\section{DISCUSSÃO}

Em relação às DCV, o presente estudo identificou que todos os trabalhadores estão na condição de sobrepeso (Tabela 1). Resultado semelhante foi encontrado em estudo anterior $^{16}$, que, ao analisarem a prevalência de fatores de risco para DCV em paramédicos, encontraram IMC $>25 \mathrm{em}$ $79 \%$ dos paramédicos. Na condição de sobrepeso pode haver um aumento de lipídeos circulantes na corrente sanguínea na forma de triglicérides e de colesterol caracterizando a dislipidemia, levando a eventos cardiovasculares ${ }^{12}$. Destaca-se, ainda, que no presente estudo $14,2 \%(n=16)$ dos trabalhadores referiu ter colesterol elevado.

A dislipidemia favorece a deposição de lipoproteína de baixa densidade nas paredes arteriais, formando lesões espessadas e endurecidas localizadas na camada íntima das artérias, denominadas de placa aterosclerótica. Essas placas podem obstruir parcialmente ou totalmente a luz das artérias, causando aumento da pressão arterial sistêmica e insuficiência do suprimento de sangue para órgãos e tecidos ${ }^{7}$. A deposição de placas juntamente com o avançar da idade, em que há o enrijecimento nas artérias, provocam aumento da resistência vascular periférica, levando a níveis pressóricos elevados, assim a combinação desses dois fatores são reconhecidamente mais suscetíveis ao desenvolvimento de HAS e de cardiopatias hipertróficas ${ }^{17}$. Neste contexto, o presente estudo encontrou associação significativa entre o IMC de sobrepeso e HAS e entre idade acima de 40 anos e HAS.

Um importante fator associado para a HAS é o estresse ocupacional ${ }^{9}$. Ainda, a sobrecarga de estímulos durante os períodos da atividade ocupacional predispõe a 
um aumento da atividade cardiovascular, provocando maior variabilidade da pressão arterial e níveis pressóricos mais elevados $^{8}$. Embora a maioria dos trabalhadores apresente exposição ao estresse ocupacional e a qualquer das variáveis que possam representá-lo, não foi encontrada associação significativa com a HAS no presente estudo.

A associação entre HAS e o tempo de serviço no SAMU 192 foi significativa. Esses achados vêm ao encontro de estudo anterior ${ }^{18}$, que em uma coorte de 15 anos, observaram que os médicos do Hospital de Clínicas de Goiás apresentavam aumento da pressão arterial, excesso de peso e dislipidemia. Porém, este dado tem relação com o aumento da idade, pois quem tem um tempo maior de serviço no SAMU 192 apresenta também um aumento da idade, o qual já foi relacionado com a HAS.

Em estudo anterior ${ }^{19}$ foi identificado nos profissionais da saúde do Hospital Universitário Federal de Santa Catarina o aparecimento de HAS, dislipidemia e estresse. Enquanto que, em enfermeiros e técnicos de enfermagem foi encontrado o estresse, sedentarismo, IMC, HAS e obesidade como fatores associados para o aparecimento de $\mathrm{DCV}^{20}$. Os resultados de todos esses estudos vêm ao encontro do presente estudo, entretanto, entre os profissionais do SAMU 192 não foi evidenciado o estresse como um aumento na chance de risco para o aparecimento da HAS.

No presente estudo, os trabalhadores referiram em sua maioria não apresentar DCV, sendo que apenas $8,8 \%$ deles informaram tal situação. Cabe comentar que os trabalhadores do presente estudo estão altamente expostos a maioria dos fatores associados ao aparecimento de DCV, os quais foram confirmados na Tabela 2, dessa forma especula-se que, em longo prazo, esses trabalhadores poderão desenvolver alguma DCV. Assim, seria necessário um acompanhamento dos hábitos de vida e ocupacionais desses trabalhadores por um período maior de tempo para detectar a ocorrência de alguma DCV.

Em relação à dor musculoesquelética nos trabalhadores do SAMU 192, a prevalência foi elevada $(92,9 \%)$, sendo que a região das "costas e MMII" foi a que apresentou maior prevalência $(35,2 \%)$ entre todas as regiões do corpo, nos últimos três meses. Esse resultado corrobora com achados anteriores ${ }^{8}$, que dentre os 930 paramédicos avaliados, $50,5 \%$ relataram de dor nas costas e MMII. Porém, no presente estudo a relação de dor e atividades laborais não foi confirmada, pois a associação não foi significativa.

A dor apresentou associação significativa com a idade, onde o trabalhador que tem 39 anos ou mais tem $6 \%$ de probabilidade a mais de ter dor do que os demais trabalhadores, confirmando os resultados de Studnek et al. ${ }^{11}$, que observaram uma tendência de aumento da dor com o avançar da idade. A primeira limitação do presente estudo é a ausência de investigação sobre o fato da população acima dos 39 anos ser também a população com maior tempo de serviço nessa atividade profissional. Mesmo assim, dentre os trabalhadores que apresentavam dor, metade apresentavam maior tempo de serviço e a outra metade não. Nesse sentido, especula-se que o tempo de serviço não parece ter influenciado o resultado encontrado.

Uma possível fonte geradora da dor musculoesquelética é o manuseio de pacientes nas mais diferentes condições, como por exemplo, a remoção de vítimas de dentro do veículo e de locais de difícil acesso com espaço físico restrito, a necessidade de conter pacientes agitados, entre outros ${ }^{12}$. Kim et al. ${ }^{21}$ ao avaliar socorristas encontrou que $70 \%$ das lesões musculoesqueléticas estão relacionadas com o manejo do paciente. O presente estudo não questionou aos trabalhadores do SAMU 192 a origem da dor musculoesquelética ou sobre suas possíveis relações. Entretanto, a exposição ao esforço físico obteve associação significativa com os DORT, sendo considerado um fator associado para o aparecimento dos DORTs (Tabela 4).

A ocorrência dos DORT está relacionada ao risco biomecânico da tarefa desenvolvida, além dos riscos psicossociais e organizacionais relacionados ao trabalho ${ }^{22}$. O risco biomecânico, contemplado nos riscos ergonômicos, compreende as adaptações e modificações musculares que o organismo necessita realizar em resposta às exigências biomecânicas da tarefa, bem como a interação entre os fatores carga de trabalho, esforço repetitivo, posturas inadequadas e vibração ${ }^{10}$. Os riscos organizacionais, também contemplados nos riscos ergonômicos, referemse aos aspectos da organização, supervisão e realização do trabalho, já os riscos psicossociais referem-se às percepções subjetivas que o trabalhador tem dos fatores organizacionais, podendo demandar pressões de natureza psicológica $^{5}$. O estresse, relacionado ao tempo e a velocidade na realização da tarefa foi identificado como um fator associado para a DORT (Tabela 3). Acredita-se que este fator esteja relacionado à: (1) demanda gerada pelo usuário, o qual consome os cuidados de saúde oferecidos pelos trabalhadores e (2) finalidade do atendimento pré-hospitalar, que é chegar precocemente à vítima após ter ocorrido um comprometimento à sua saúde e dar a devida resolubilidade ao caso ${ }^{23}$. Gallagher e McGilloway ${ }^{24}$ afirmam que a pressão de tempo para a realização da tarefa pode ter efeito sobre a saúde física e mental dos trabalhadores.

Cabe salientar que é relevante a influência que a satisfação no trabalho parece exercer sobre os trabalhadores. A insatisfação pode ser decorrente de sentimentos 
de indignidade pela obrigação de realizar uma tarefa desinteressante e sem significado, de inutilidade por desconhecer o que representa o trabalho no conjunto das atividades na empresa, de desqualificação, tanto em função de questões salariais como ligadas à valorização do trabalho, em aspectos como responsabilidade, risco ou conhecimentos necessários ${ }^{6}$. Portanto, a saúde do trabalhador diz respeito à relação congruente entre a estrutura, o funcionamento das organizações e o bem-estar das pessoas que nelas trabalham ${ }^{22}$.

Ainda, outra variável que se comportou como risco para as DORT foi o regime de trabalho, pois quem realiza plantão de 12 horas tem $15 \%$ de chances a mais de ter DORT que os demais trabalhadores. Além disso, o aumento nas horas trabalhadas impacta na produtividade, por exemplo, foi relatado em estudo anterior ${ }^{25}$ que o aumento da carga horária para mais que 8 horas diárias diminui a produtividade do trabalhador. Entretanto, embora no presente estudo não tenha sido avaliado a produtividade dos trabalhadores do SAMU 192, mesmo assim salienta-se a necessidade de ver essa questão com cautela e cuidado. Ainda, o presente estudo identificou que a falta de pausa nas atividades constitui também um fator associado com a DORT (Tabela 4). Broniecki et al. ${ }^{26}$ ao avaliarem os fatores associados às lesões musculoesqueléticas identificaram que os trabalhadores de ambulância foram mais propensos a apresentar lesão, pois não eram capazes de fazer pausas suficientes durante o trabalho.

As DORT referidas pelos trabalhadores do SAMU 192 foram tendinite, bursite, tenossinovite e hérnia de disco. Especula-se que a tendinite no punho referida por $39 \%$ dos trabalhadores pode ser decorrência da vibração excessiva do motor e da velocidade dos veículos utilizados para emergências, do esforço físico e da ausência de pausa entre os condutores e técnicos, por exemplo. Essa realidade brasileira poderá em médio prazo impactar negativamente o Sistema Único de Saúde, e deve ser considerada na proposição de ações que minimizem esses riscos inerentes à profissão para esses trabalhadores.

Uma segunda limitação do presente estudo é o fato de que as condições de saúde foram informadas pelos participantes e não obtidas por meio de exames laboratoriais e/ou avaliações médicas, o que pode ter prejudicado os resultados referentes a DCV. Entretanto, em pesquisas dessa natureza, é comum a obtenção de informações a partir do auto relato dos investigados. Não obstante, o uso do questionário como única fonte de coleta de dados constitui-se de fato em uma limitação, uma vez que o preenchimento individual dos questionários e a não comunicação entre os participantes não foram controlados; $\mathrm{e}$ o questionário não utilizou escalas adequadas para avaliação do estresse, bem como as desordens de cunho mental foram informadas em uma questão aberta, não sendo oferecidas opções dos diferentes tipos de desordens mentais para que o trabalhador pudesse assinalar. Uma opção interessante para contornar essa limitação seria associar ao uso do questionário QHT a condução de entrevistas individuais e/ou a inclusão de questões abertas e descritivas. Essa estratégia auxiliaria no esclarecimento de alguns dos fatores relacionados aos aspectos organizacionais e psicossociais, mas principalmente, forneceria informação para o entendimento das causas e possibilidades de modificação dos fatores nocivos à saúde dos trabalhadores, bem como nas estratégias utilizadas por eles para manterem-se saudáveis. Ainda, uma terceira limitação do presente estudo compreende a falta de informações complementares sobre os aspectos organizacionais e psicodinâmicos do trabalho, visto que parecem ser um dos riscos ocupacionais que mais influenciam a saúde dos trabalhadores da SAMU 192.

Por fim, é importante apontar para a necessidade de mais estudos e de aprofundamento dos aspectos nocivos relacionados ao trabalho nos trabalhadores do SAMU 192, pois estudos dessa natureza podem subsidiar os serviços de saúde nas ações de intervenção. Dessa forma, estudos que identifiquem a prevalência e os agentes nocivos relacionados ao trabalho apontam para um campo rico a ser estudado.

A formulação das estratégias de intervenções se inicia com uma análise ergonômica do trabalho, por meio de um processo construtivo e participativo para a resolução de problemas complexos; uma análise da população de trabalhadores; e uma análise referente a queixas e insatisfações ${ }^{5}$. Os resultados do presente estudo demonstraram a prevalência de dor, DORT e DCV e os fatores associados que influenciam a saúde do trabalhador do SAMU 192, contribuindo tanto para o fomento de ações de intervenção, quanto para o debate acadêmico. Esses resultados devem auxiliar no conhecimento da realidade desses profissionais e na compreensão de suas insatisfações, requisitos iniciais na busca dos meios de transformação desse trabalho.

Nesse contexto, entende-se que a partir dessa avaliação inicial, dada pelo resultado do presente estudo, a coordenação geral do serviço pré-hospitalar do SAMU 192, em ação conjunta com a Secretaria Municipal de Saúde, poderá pensar em intervenções para auxiliar o funcionamento da equipe. Ou seja, ações voltadas para discutir a organização do trabalho e a satisfação no trabalho poderão ser implementadas, evitando novos casos e agravos dos quadros já instalados, tais como: espaços de discussão, terapias individuais e coletivas, ginástica laboral, formação 
e qualificação profissional quanto aos riscos ocupacionais e suas prevenções, revisão da carga de trabalho de cada trabalhador, entre outras. Além disso, fica evidente a necessidade de transformação nas condições e organização do trabalho e em todos os aspectos que estão contribuindo para o adoecimento dessa população. Cabe comentar que os resultados desse estudo já foram apresentados aos trabalhadores em cada uma das bases descentralizadas, bem como à gerência e equipe do SAMU 192, como uma ação inicial de promoção da saúde.

\section{CONCLUSÃO}

Conclui-se que os profissionais do SAMU 192 Porto Alegre/RS apresentaram alta prevalência de dor $(92,9 \%)$ e DORT $(50,4 \%)$, e baixa prevalência de DCV $(8,8 \%)$. A

\section{REFERÊNCIAS}

1. Soerensen AA, Moriya TM, Soerensen R, Robazzi MLDCC. Atendimento pré-hospitalar móvel: fatores de riscos ocupacionais. Rev Enferm UERJ. 2008;16:187-92. Disponível em: http://www.facenf.uerj.br/v16n2/v16n2a08.pdf.

2. Brasil. Ministério da Saúde. Política nacional de atenção às urgências. 3a ed. Brasília: Ministério da Saúde; 2006. Disponível em: http://bvsms.saude.gov.br/bvs/publicacoes/ politica_nacional_atencao_urgencias_3ed.pdf.

3. Brasil. Ministério da Saúde. Rede Nacional SAMU 192. Brasília: Ministério da Saúde; 2012.

4. Sousa ATO, Souza ER, Costa ICP. Riscos ocupacionais no atendimento pré-hospitalar móvel: produção científica em periódicos online. Rev Bras Ciên Saúde. 2014;18(2):167-74. doi: 10.4034/RBCS.2014.18.02.12.

5. Brasil. Ministério do Trabalho e Emprego. Manual de aplicação da Norma Regulamentadora ${ }^{\circ}$ 17. 2a ed. Brasília: Ministério do Trabalho e Emprego, Secretaria de Inspeção do Trabalho; 2002. Disponível em: http://www.ergonomia.ufpr. br/MANUAL_NR_17.pdf.

6. Martinez MC, Paraguay AIBB. Satisfação e saúde no trabalho - aspectos conceituais e metodológicos. Cad Psicol Soc Trab. 2003;6:59-78. doi: 10.11606/issn.1981-0490.v6i0p59-78.

7. Nusshold P. Organização do trabalho e sofrimento psíquico nas atividades de serviço. O caso dos call centers na Argentina. Laboreal. 2017;13(1):81-5. doi: 10.15667/LABOREALXIII0117PN

8. Couto HA, Vieira FLH, Lima EG. Estresse ocupacional e hipertensão arterial sistêmica. Rev Bras Hipertens. 2007;14(2):112-5. Disponível em: http://departamentos. cardiol.br/dha/revista/14-2/11-ocupacional.pdf. idade atuou como fator associado ao aparecimento da dor. Os riscos associados ao aparecimento da DORT foram: físico (exposição ao frio, calor e vibrações), ergonômico (esforço físico, ausência de pausa nas atividades, profissão e regime de trabalho) e o estresse.

A associação entre os fatores associados e DCV não foi possível devido à baixa prevalência de DCV entre os trabalhadores. Considerando as associações entre os próprios fatores associados, a HAS apresentou associação significativa com o IMC, a idade e o tempo de serviço. Ainda, foram considerados fatores associados ao estresse: a profissão (condutor de ambulância e técnico de enfermagem), todas as variáveis que envolvem pressão para a produtividade e todas as variáveis que envolvem restrição do sono. Não obstante, cabe salientar que o estudo sobre os riscos e os fatores associados que contribuem para o adoecimento do SAMU 192 carece de mais aprofundamento.

9. Cordeiro R, Fischer FM, Lima Filho EC, Moreira Filho DC. Ocupação e hipertensão. Rev Saúde Pública. 1993;27:380-7. doi: 10.1590/S0034-89101993000500010.

10. Brasil. Ministério do Trabalho. Organização Pan-Americana da Saúde no Brasil. Doenças relacionadas ao trabalho: manual de procedimentos para os serviços de saúde. Brasília: Ministério da Saúde; 2001. Disponível em: http://bvsms.saude.gov.br/bvs/ publicacoes/doencas_relacionadas_trabalho1.pdf.

11. Studnek JR, Crawford JM, Wilkins Jr RD, Pennell ML. Back problems among emergency medical services professionals: the LEADS health and wellness follow-up study. Am J Ind Med. 2010;53(1):12-22. doi: 10.1002/ajim.20783.

12. Célia RCRS, Alexandre NMC. Distúrbios osteomusculares e qualidade de vida em trabalhadores envolvidos com transporte de pacientes. Rev Bras Enferm. 2003;56:494-8. doi: 10.1590/ S0034-71672003000500005.

13. Santos Filho RD, Martinez TLR. Fatores de risco para doença cardiovascular: velhos e novos fatores de risco, velhos problemas! Arq Bras Endocrinol Metab. 2002;46(3):212-4. doi: 10.1590/S0004-27302002000300002.

14. Lúcio MG, Gusmão CMP, Torres MC. Riscos ocupacionais do atendimento pré-hospitalar: uma revisão bibliográfica. Interfaces Cient. 2013;1(3):69-77. doi: 10.17564/2316-3798.2013v1 n3p69-77.

15. Chaise FO, Kasten AP, Furlanetto TS, Pasa J, Candotti CT. Validade e reprodutibilidade do Questionário de Hábitos relacionados ao Trabalho (QHT) para trabalhadores do SAMU 192. Rev Ter Ocup Univ São Paulo. 2016;27(2):199-215. doi: 10.11606/issn.2238-6149.v27i2p199-215.

16. Barret TW, Norton VC, Busam M, Boyd J, Maron DJ, 
Slovis CM. Self-reported cardiac risk factors in emergency department nurses and paramedics. Prehosp Disaster Med. 2000;15(2):14-7. doi: 10.1017/S1049023X00025036.

17. Sant'anna MP, Montenegro LT, Araújo MM. Hipertrofia cardíaca esquerda e direita em necropsias de hipertensos. Rev Assoc Med Bras. 2012;58(1):41-7. doi: 10.1590/S010442302012000100013.

18. Jardim TDSV, Jardim PCSBOV, Araújo WECD, Jardim LMSSV, Salgado CM. Fatores de risco cardiovasculares em coorte de profissionais da área médica: 15 anos de evolução. Arq Bras Cardiol. 2010;95:332-8. doi: 10.1590/S0066782X2010005000084.

19. Cantos GA, Duarte MDFS, Dutra RL, Silva CDSMD, Waltrick CDDA, Balen MDG, et al. Prevalência de fatores de risco de doença arterial coronária em funcionários de hospital universitário e sua correlação com estresse psicológico. J Bras Patol Med Lab. 2004;40:240-7. doi: 10.1590/S167624442004000400006.

20. Bottoli C, Moraes MA, Goldmeier S. Fatores de risco cardiovasculares em trabalhadores de enfermagem em um centro de referência no sul do Brasil. Cien Enferm. 2009;15:101-9. doi: 10.4067/S0717-95532009000300011.

21. Kim H, Dropkin J, Spaeth K, Smith F, Moline J. Patient handling and musculoskeletal disorders among hospital workers: analysis of 7 years of institutional workers' compensation claims data. Am J Ind Med. 2012;55(8):683-90. doi: 10.1002/ajim.22006.

22. Peçanha DLN. Cultura organizacional e saúde - contribuições da psicodinâmica do trabalho. Bol Acad Paul Psicol. 2009;77(2/9):329-44. Disponível em: http://pepsic. bvsalud.org/scielo.php?script $=$ sci_arttext\&pid=S1415711X2009000200010.

23. Wiebbelling ED, Santos MF. Enfermagem em urgência e emergência no município de Foz do Iguaçu, Paraná, Brasil. Rev Enferm UFPE. 2009;3(3):1-10. doi: 10.5205/01012007.

24. Gallagher S, McGilloway S. Experience of critical incident stress among ambulance service staff and relationship to psychological symptoms. Int J Emerg Ment Health. 2009;11(4):235-48. doi: 10.1136/emj.2009.080622.

25. Grandjean E. Manual de ergonomia: adaptando o trabalho ao homem. Porto Alegre: Bookman; 1998.

26. Broniecki M, Esterman A, Grantham H. Risk factors for back, neck and shoulder musculoskeletal injuries and claims in ambulance officers. J Musculoskelet Res. 2015;15(1):1250009. doi: 10.1142/S0218957712500091. 